with zero impact parameter, and with the diatom vibrational quantum number, $v$, initially set equal to $-1 / 2$ to suppress vibration in the molecule. These results showed the same trends as the $3 \mathrm{D}$ calculations. The model, using a potential energy function similar to the LSTH near the saddlepoint, was able to reproduce these trends successfully. Near threshold $P^{r}(j)$ for low $j$ may increase slowly, or even decrease, since motion in the $\gamma$ coordinate tends to deflect the trajectory away from the saddlepoint. At higher values of $j$, however, the extra energy available due to the rotation makes more of the valley in the saddlepoint region of the bend potential energetically accessible, and reaction occurs readily. For higher translational energies, the ability of the potential to deflect trajectories is reduced, and increase in rotation always enhances reactivity. These trends have been seen in 3D studies for $\mathrm{H}+$ $\mathrm{H}_{2}{ }^{6}$ and $\mathrm{H}+\mathrm{Cl}_{2}{ }^{8}$
It was found to be necessary that the moment of inertia of the target diatom increase as the saddlepoint is approached. This led us to predict that rotation would enhance reactivity most effectively in systems with a late barrier to reaction.

The results were found to be sensitive to changes in the bending potential. A softer bend obviously allows reaction to occur more easily. More interestingly, the results were found to be sensitive to the long-range anisotropy of the bending potential. It therefore seems possible that rotationally excited reactants could be used to probe this part of the potential surface.

Acknowledgment. Acknowledgment is made to the donors of the Petroleum Research Fund, administered by the American Chemical Society, for support to H.R.M. of this research.

Registry No. H, 12385-13-6; $\mathrm{H}_{2}, 1333-74-0$.

\title{
Excited-State Intramolecular Proton Transfer in Jet-Cooled 2,5-Bis(2-benzothiazolyl)hydroquinone
}

\author{
N. P. Ernsting, ${ }^{*}$ A. Mordziñski, ${ }^{\dagger}$ and B. Dick \\ Max-Planck-Institut für biophysikalische Chemie, Abteilung Laserphysik, D-3400 Göttingen, \\ Federal Republic of Germany (Received: August 26, 1986)
}

\begin{abstract}
The fluorescence of 2,5-bis(benzothiazolyl)hydroquinone was studied for isolated molecules cooled in a supersonic free neon jet. It consists of a strongly Stokes-shifted fluorescence band, and it is assigned to the molecule which is formed by excited-state intramolecular proton transfer. The fluorescence excitation spectrum shows a progression in a $114-\mathrm{cm}^{-1}$ vibrational mode of the excited state. The observed spectrum is more congested than that of the parent oxazole under otherwise identical experimental conditions. It is concluded that the thiazole has no significant intrinsic barrier to excited-state intramolecular proton transfer. Weak vibronic bands were observed by fluorescence excitation in the adjoining long-wavelength spectral region. They could indicate "nonvertical" transitions to the proton-transferred excited molecule.
\end{abstract}

\section{Introduction}

Aromatic molecules having a phenolic hydroxyl group with an intramolecular hydrogen bond to a nearby heteroatom of the same chromophore often show fluorescence with an anomalously large Stokes shift. This fluorescence arises from the product of an excited-state reaction, namely excited-state intramolecular proton transfer (ESIPT; for example, see Figure 1). The renewed interest in ESIPT ${ }^{1-3}$ concerns mainly the mechanism and rates of the reaction in solution. It was shown that ESIPT usually has rates greater than $10^{11} \mathrm{~s}^{-1}$, independent of $H / D$ exchange. Some investigators suggest that the reaction occurs partly from the hot, vibrationally unrelaxed molecules. 4,5 The established conclusion is that ESIPT has no significant energy barrier.

The reaction in solution may be complicated by specific $\mathrm{H}$ bonding solvation or by the existence of distinct conformers having different internal $\mathrm{H}$-bonding sites. Thus it is often difficult to separate the intrinsic ESIPT reaction from solvent effects. ${ }^{6,7}$ Some of these problems may be overcome by the study of cold, isolated molecules in supersonic jets or in noble gas matrices. Fluorescence excitation studies of methyl salicylate $e^{8-10}$ and 1,5-dihydroxyanthraquinone ${ }^{11}$ have shown three main results. First, the intensity of the electronic origin band is relatively low, and long FranckCondon progressions involving a low-frequency mode of the excited state are found. Second, a strong increase of fluorescence intensity and spectral density is observed if the excess vibrational excitation energy is increased above some threshold value. Third, the fluorescence lifetimes are identical across the entire emission spectrum, and the rise time of the tautomer fluorescence could

\footnotetext{
${ }^{\dagger}$ On leave from the Institute of Physical Chemistry of the Polish Academy
} of Sciences, 01-224 Warsaw, Poland. not be resolved when excitation pulses with duration of $15 \mathrm{ps}$ were used. It was concluded that the absorption of a photon leads directly to an adiabatic excited state which is formed by a strong $\mathrm{H}$-bonding interaction between the original and proton-transferred excited forms. ${ }^{8}$

A different situation exists for 2,5-bis(2-benzoxazolyl)hydroquinone (Figure 1, with both $\mathrm{S}$ exchanged for $\mathrm{O}$ ). A blue fluorescence band and a red fluorescence band were observed in solution. ${ }^{12,13}$ Contrary to previous cases, a marked dual fluorescence was also found for the jet-cooled molecule excited near the electronic origin. ${ }^{14}$ The ratio of the quantum yield for red fluorescence to the quantum yield for blue fluorescence de-

(1) Klöpffer, W. Adv. Photochem. 1977, 10, 317.

(2) Huppert, D.; Gutman, M.; Kaufmann, K. J. Adv. Chem. Phys. 1981, 47 , part 2,643 .

(3) Flom, S. R.; Barbara, P. F. J. Phys. Chem. 1985, 89, 4489.

(4) Barbara, P. F.; Rentzepis, P. M.; Brus, L. E. J. Am. Chem. Soc. 1980 102,2786

(5) Barbara, P. F.; Brus, L. E.; Rentzepis, P. M. J. Am. Chem. Soc. 1980, 102,5631

(6) Sandros, K. Acta Chem. Scand., Ser. A 1976, 30A, 761.

(7) McMorrow, D.; Kasha, M. J. Phys. Chem. 1984, 88, 2235.

(8) Goodman, J.; Brus. L. E. J. Am. Chem. Soc. 1978, 100, 7472.

(9) Felker, P. M.; Lambert, Wm. R.; Zewail, A. H. J. Chem. Phys. 1982. $77,1603$.

(10) Heimbrook, L. A.; Kenny, J. E.; Kohler, B. E.; Scott, G. W. J. Phys. Chem. 1983, 87, 280.

(11) Van Benthem, M. H.; Gillispie, G. D. J. Phys. Chem. 1984, 88, 295.

(12) Mordzinski, A.: Grabowska, A.; Kühnle, W.; Krowczynski, A. Chem. Phys, Lett. 1983, 101, 291

(13) Mordzinski, A.; Grabowska, A.; Teuchner, K. Chem. Phys. Lett. 1984, 111, 383 .

(14) Ernsting, N. P. J. Phys. Chem. 1985, 89, 4932. 


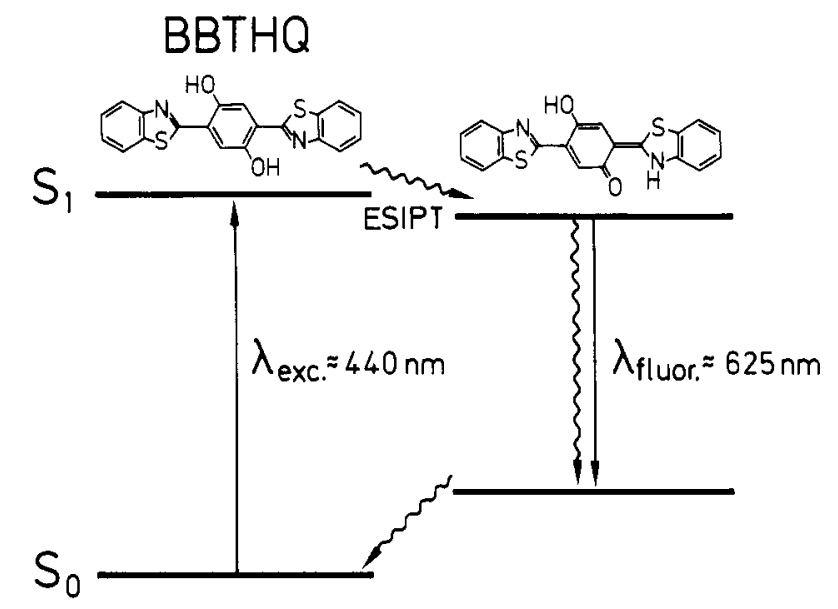

Figure 1. Excited-state intramolecular proton transfer in BBTHQ.

pends on $H / D$ substitution and increases strongly with excess vibrational energy, indicating a significant energy barrier to ESIPT.

Replacement of oxygen by sulfur in the heteroatomic parts of the chromophore gives 2,5-bis(2-benzothiazolyl)hydroquinone (BBTHQ, Figure 1). The substitution should widen the heteroaromatic frame, which would in turn decrease the $\mathrm{OH} \cdots \mathrm{N}$ distance. Both steric and electronic changes should affect the strength of the intramolecular $\mathrm{H}$ bond and the rate for excited-state intramolecular proton transfer. In this paper we examine the fluorescence excitation spectrum of jet-cooled BBTHQ and compare the results with those of its parent compound.

\section{Experimental Section}

BBTHQ was synthesized as in ref 12 . It was purified by repeated crystallization from DMF followed by sublimation. Mass spectroscopy revealed two impurities with a molecular weight of 390 and 419 . At a sample temperature of $523 \mathrm{~K}$ corresponding to the sample temperature used in the jet experiments, the impurity partial vapor pressures were determined from the mass spectrum to be $0.6 \%$ and $0.3 \%$ of the BBTHQ partial pressure, respectively. Further purification proved extremely difficult because of the low solubility of the compound in all solvents. The spectroscopic results will be discussed while keeping the existence of impurities in mind.

For survey spectra, the BBTHQ sample was sublimated at 453 $\mathrm{K}$ together with an argon stream onto a sapphire window maintained at $20 \mathrm{~K}$. The fluorescence spectra of the sample in the solid argon host are shown in Figure 2.

A continuous jet was formed by a glass nozzle of $130 \mu \mathrm{m}$ diameter at $610 \mathrm{~K}$, with a neon stagnation pressure of 500 Torr. The formation of molecular van der Waals complexes with neon is negligible under these conditions. Fluorescence was excited 2.8 $\mathrm{mm}$ downstream from the nozzle by a pulsed tunable dye laser. It was monitored through various optical cutoff filters and interference filters in order to determine the contribution of fluorescence with normal Stokes shift to the total fluorescence intensity. Partial deuteriation of the sample was achieved by the addition of 4.6 Torr of $\mathrm{D}_{2} \mathrm{O}$ vapor to the expansion mixture. Since the addition of water might change the cooling properties of the supersonic beam and thus enhance spectral sequence bands, the experiment was repeated with $\mathrm{H}_{2} \mathrm{O}$ vapor; the spectral changes were found to be insignificant in this case.

\section{Results}

The fluorescence spectrum of BBTHQ in a solid argon host (Figure 2) has a dominant fluorescence band at $625 \mathrm{~nm}$. Its large Stokes shift of ca. $6700 \mathrm{~cm}^{-1}$ indicates that excited-state intramolecular proton transfer has occurred and that the emission emanates from the excited tautomeric molecule (cf. Figure 1). A relatively weak emission with a normal Stokes shift has an excitation spectrum which differs from that of the dominant fluorescence. It may be due to BBTHQ molecules in which ESIPT is inhibited, either because the molecular conformation prior to deposition did not allow an intramolecular $\mathrm{OH}$... $\mathrm{N}$ hydrogen bond (rotamer), or because the local environment of the argon host subtly disturbs the geometry which is necessary for the excitedstate reaction.

For isolated, jet-cooled BBTHQ, only the strongly Stokes-shifted fluorescence could be detected. The corresponding fluorescence excitation spectrum is shown in Figure 3.

The first vibronic band was observed at $461.47 \mathrm{~nm}$, or 21664 $\mathrm{cm}^{-1}$. A search at longer wavelengths revealed only a weak band shifted by $-123 \mathrm{~cm}^{-1}$. Its relative intensity decreased with better cooling through increased stagnation pressure, indicating a vibrational hot band. Therefore the band at $461.47 \mathrm{~nm}$ is assigned

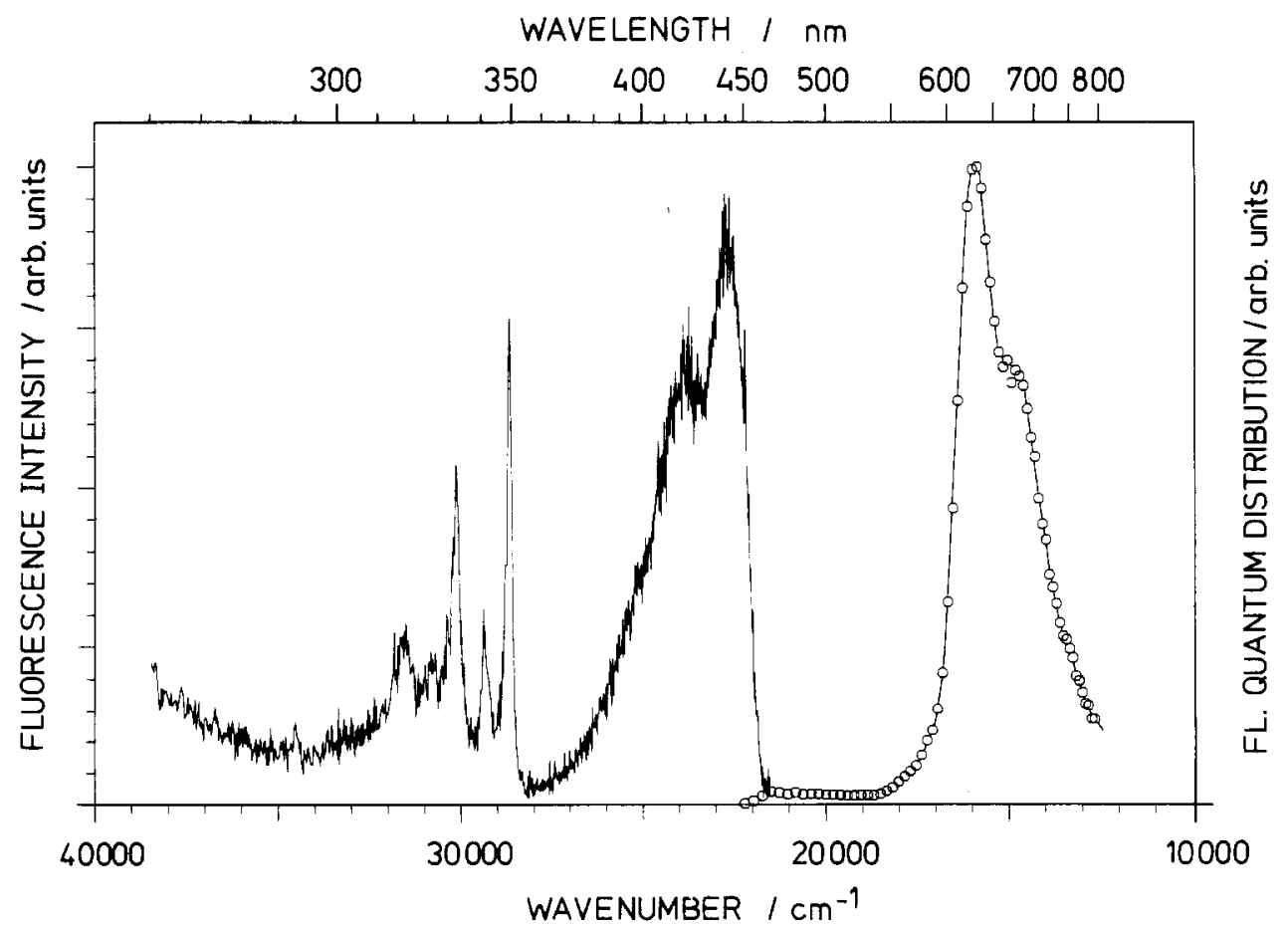

Figure 2. Fluorescence spectra of BBTHQ in a solid argon host at $20 \mathrm{~K}$. The left trace is an excitation spectrum of fluorescence with $\lambda_{F}=650 \pm$ $2 \mathrm{~nm}$. The right trace is the wavelength-resolved emission spectrum, from excitation at $415 \mathrm{~nm}$ (measured at wavelength intervals of $5 \mathrm{~nm}$ with a bandwidth of $4 \mathrm{~nm}$ ). 


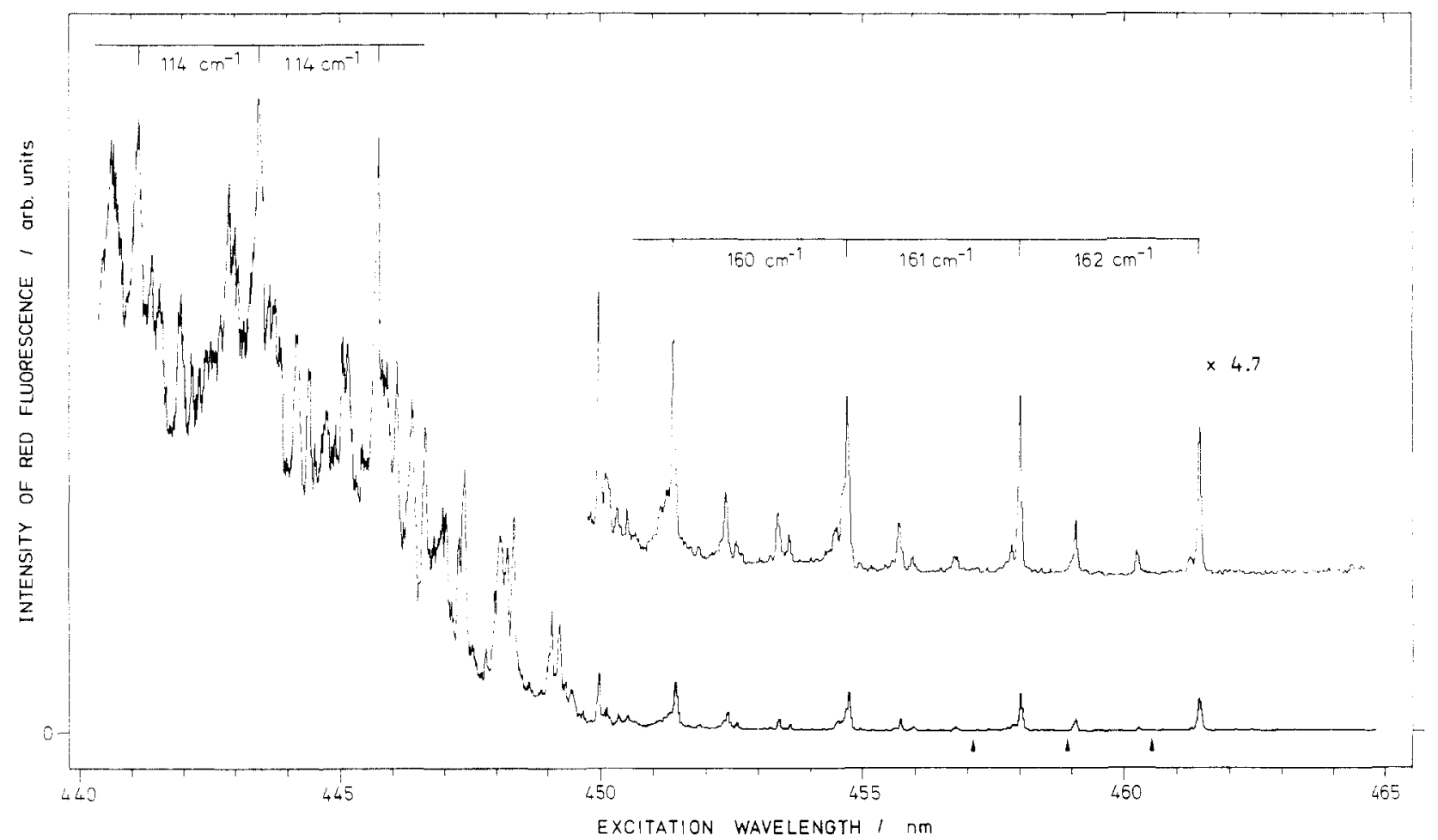

Figure 3. Excitation spectrum of red fluorescence $\left(\lambda_{F} \geq 580 \mathrm{~nm}\right)$ for the jet-cooled BBTHQ sample. Vibronic progressions are indicated, and arrows mark the positions of the first three $d$ bands.

to a vibronic origin transition and other bands will be denoted by their relative excitation energies. A clear vibronic progression extends from the reference origin band to higher energies, with comparable intensities and vibrational spacing of ca. $162 \mathrm{~cm}^{-1}$. Similar progressions are also based on weaker bands at 56 and $110 \mathrm{~cm}^{-1}$.

With excess excitation energies above $550 \mathrm{~cm}^{-1}$, a marked spectral change is apparent from Figure 3. Many new bands appear; the intensity of the resolved bands increases sharply, and spectral congestion by cold bands (and possibly by associated sequence band due to insufficient cooling of this large molecule) leads to an unresolved background for fluorescence excitation.

The first prominent band occurs at relative excitation energy of $771 \mathrm{~cm}^{-1}$, and it is up to 16 times more intense than the reference origin band. Together with other prominent bands at 885 and $998 \mathrm{~cm}^{-1}$, it clearly starts a progression with a vibrational spacing of $114 \mathrm{~cm}^{-1}$. Indeed that spacing is ubiquitous here so that the spectrum above $680 \mathrm{~cm}^{-1}$ can largely be decomposed into three similar band clusters around and including each prominent band.

The fluorescence excitation spectrum of a partly deuteriated sample was examined with relative excitation energy up to 215 $\mathrm{cm}^{-1}$ only. Partial deuteriation caused the appearance of new sharp bands at 44,157 , and $206(=162+44) \mathrm{cm}^{-1}$ (cf. Figure 2), with relative intensities of $\mathrm{ca}$. 1:0.6:1.

\section{Discussion}

The Short-Wavelength Part of the Excitation Spectrum. The observed fluorescence excitation spectrum shown in Figure 3 is readily compared to the corresponding spectrum for the parent compound 2,5-bis(2-benzoxazolyl)hydroquinone, BBXHQ. ${ }^{14}$ The comparison shows that the intense, short-wavelength part of the spectrum in Figure 3 (with $\lambda \leq 447.3 \mathrm{~nm}$ ) may be matched to the excitation spectrum of red fluorescence from jet-cooled BBXHQ. The latter spectrum was characterized by groups of vibronic bands centered around a prominent band, the groups being arranged in a progression of a $115-\mathrm{cm}^{-1}$ vibrational, skeletal mode of the excited state. Returning to Figure 3, the first three prominent bands occur at wavelengths of 445.61, 443.35, and $441.14 \mathrm{~nm}$, with a vibrational spacing of $114 \mathrm{~cm}^{-1}$. However, the weaker bands which cluster around a prominent band have greatly increased in number by comparison. No significant blue fluorescence of normal Stokes shift was detected from the jetcooled sample for any excitation wavelength. This is in marked contrast to isolated, jet-cooled BBXHQ which fluoresces mostly blue if excited at the first prominent vibronic band, indicating a barrier to excited-state intramolecular proton transfer. ${ }^{14}$

We conclude: The intense, short-wavelength part of the spectrum in Figure 2 can be assigned to BBTHQ because of its similarity to the spectrum of the parent oxazole, and because its relatively high intensity points to the main sample compound. The lack of normal fluorescence shows that BBTHQ has no significant intrinsic barrier to an exothermic ESIPT reaction. Near-vertical excitation of the thiazole in its ground-state hydroquinone form should therefore lead to higher vibrational states of the electronically excited adiabatic state which encompasses both tautomeric forms along some appropriate proton-transfer coordinate. The vertical Franck-Condon vibronic states should be mixed into a relatively dense background of vibronic states belonging to the electronically excited adiabatic state. This is borne out by the observation that the characteristic spectrum of the thiazole (short-wavelength part of Figure 2) has higher spectral density than the corresponding spectrum of the oxazole BBXHQ recorded under otherwise identical recording conditions.

Comparison with Other Molecules. Similar conclusions have been drawn for methyl salicylate ${ }^{8}$ and 1,5-dihydroxyanthraquinone. ${ }^{11}$ The fluorescence excitation spectrum of methyl salicylate in a solid neon host shows a progression of marked intensity thresholds with increasing excitation energy. These appear to be a Franck-Condon progression in an excited-state mode in the $350-\mathrm{cm}^{-1}$ range, and the $(0,0)$ origin could be identified. The extreme intensity increase for each successive member indicates that a large displacement has occurred along the appropriate normal coordinate. Hence vertical excitation leads to higher vibrational levels of the electronically excited state.

The fluorescence excitation spectrum of 1,5-dihydroxyanthraquinone in Shpol'skii matrices ${ }^{11}$ is somewhat different in character. The first $1000 \mathrm{~cm}^{-1}$ of the excitation spectrum is structured and reasonably regular. It is followed by a region of greater intensity and spectral scrambling. The observed intensity rise is less pronounced for the isolated, jet-cooled molecule ${ }^{11}$ probably because the vibrational energy remaining after electronic excitation would enhance nonradiative deactivation and result in loss of monitored fluorescence yield. The results were discussed by assuming an 
asymmetric double-well potential for the excited state along a coordinate for the transfer of a single proton. The intense, short-wavelength part of the excitation spectrum was assigned to (near-)vertical transitions to excited 1,5-dihydroxyanthraquinone in its original 9,10-quinone form. Excited-state intramolecular proton transfer would then lead to an excited tautomer at lower energy (by ca. $1100 \mathrm{~cm}^{-1}$ ) with a 1,10 -quinone-like structure and, in effect, to widely Stokes-shifted fluorescence. The weak and regular long-wavelength part of the excitation spectrum was attributed to transitions from the ground-state 9,10-quinone directly to the excited 1,10-quinone-like, proton-transferred tautomer.

The Long-Wavelength Part of the Excitation Spectrum. We return to jet-cooled 2,5-bis(2-benzothiazolyl)hydroquinone. The fluorescence excitation spectrum also shows a weak long-wavelength part, with an origin at $461.47 \mathrm{~nm}$ and a clear progression with vibrational spacing of $162 \mathrm{~cm}^{-1}$. This long-wavelength spectrum may have been caused by the sample impurities mentioned earlier. However, it is instructive to speculate briefly on an alternative explanation which follows the hypothesis proposed for 1,5-dihydroxyanthraquinone. Assume that the long-wavelength spectrum indicates transitions which lead from the original, hydroquinone form of BBTHQ in the ground state directly-albeit with small Franck-Condon factors - to a region of the excited-state potential energy surface which may be associated with the proton-transferred tautomer. There are two observations which support this assumption.

First, a hot band was observed shifted by $123 \mathrm{~cm}^{-1}$ to the red from the origin band, while the vibronic progressions in this part of the spectrum are caused by a $162-\mathrm{cm}^{-1}$ vibrational mode of the excited state. A ground-state vibrational mode of $123 \mathrm{~cm}^{-1}$ was also found to be optically active for the parent oxazole compound in its original, hydroquinone form. ${ }^{14}$ The similarity suggests that the long-wavelength origin band in the spectrum of Figure 2 likewise marks a transition which emanates from BBTHQ in its hydroquinoid ground state. The increase of vibrational frequency upon electronic excitation is unusual, and it is likely that the underlying increase of skeletal rigidity is connected to an altered electronic structure as a result of ESIPT. This is consistent with the assumption that the $461.47-\mathrm{nm}$ origin band indicates a weak, "nonvertical" electronic transition from BBTHQ in its hydroquinone ground state directly to the excited proton-transferred form.

Second, partial deuteriation of the BBTHQ sample resulted in two new origin bands blue-shifted by 44 and $157 \mathrm{~cm}^{-1}$, respectively, from the reference origin for the undeuteriated sample. Similar blue shifts have been observed for other molecules with ESIPT; ${ }^{10,11,14}$ a shift to shorter wavelength upon $\mathrm{OH} / \mathrm{OD}$ exchange indicates that the intramolecular hydrogen bond becomes stronger with electronic excitation. ${ }^{15}$ For the parent oxazole BBXHQ, deuterium substitution led to two new origin bands with blue shifts of $57 \mathrm{~cm}^{-1}$. They were attributed to BBXHQ- $d_{1}$ and $-d_{2}$. As the shift induced per $\mathrm{H} / \mathrm{D}$ exchange is identical for $\mathrm{BBXHQ}-d_{1}$ and $-d_{2}$, it was concluded that the ground and excited states have two equivalent intramolecular hydrogen bonds in this case. ${ }^{14}$ For the thiazole BBTHQ, the observed blue shifts are not related in the same manner, indicating that two inequivalent hydrogen bonds exist in the ground or excited electronic state, or both. It is reasonable to assume that only a single proton is transferred in the excited state of BBTHQ, as was found for the parent oxazole BBXHQ. ${ }^{12}$ The excited tautomeric form of BBTHQ should then have two inequivalent hydrogen bonds (cf. Figure 1). If optical excitation can indeed lead directly to the excited tautomeric form, then single $H / D$ substitution should already result in two different new origin bands, depending on whether the deuteron carries out ESIPT or not. It is tempting to associate the d band which is moderately shifted by $44 \mathrm{~cm}^{-1}$ with an excited $\geq N$...D-O...= $\mathrm{O} \cdot . \mathrm{H}-\mathrm{N}<$ structure, and the $\mathrm{d}$ band shifted by $157 \mathrm{~cm}^{-1}$ with $\geq \mathrm{N} . . \cdot \mathrm{H}-\mathrm{O}-. .=\mathrm{O} \cdot . \mathrm{D}-\mathrm{N}<$. Further experimental work is needed to clarify the carrier and nature of the long-wave spectral part of Figure 3.

\section{Conclusion}

The fluorescence excitation spectrum of jet-cooled 2,5-bis(benzothiazolyl)hydroquinone (BBTHQ) has been observed and compared to that of the parent oxazole compound. In both cases, the spectra are characterized by a progression in a ca. $114-\mathrm{cm}^{-1}$ vibrational mode of the excited state. The spectrum of the thiazole is more congested than that of the oxazole under identical conditions. No significant blue fluorescence was observed for the thiazole, in contrast to previous results for the oxazole. It was concluded that (near-)vertical excitation, at wavelengths below $450 \mathrm{~nm}$, leads to higher vibrational levels of an electronically excited state which has no significant barrier to intramolecular proton transfer.

Acknowledgment. We are grateful to Dr. U. Brackmann (Lambda Physik) for the synthesis of BBTHQ, to Dr. W. Kühnle (Abteilung Spektroskopie) for MS analysis, and to Prof. F. P. Schäfer and the Deutsche Forschungsgemeinschaft for their support of this work.

Registry No. 2,5-Bis(2-benzothiazolyl)hydroquinone, 33450-09-8.

(15) Smulevich, G.; Amirav, A.; Even, U.; Jortner, J. Chem. Phys. 1982, 